\title{
Investigation of the torsional deformation of the complete upper denture: A pilot study
}

\author{
Anthony E. Prombonas ${ }^{1}$, Maria A. Paralika ${ }^{2}$, Nikolas A. Poulis ${ }^{1}$ \\ ${ }^{1}$ Department of Dental Technology, Technological and Educational Institute of Athens, Athens, Greece \\ ${ }^{2}$ Department of Mechanical Engineering and Civil Work, Technological and Educational Institute of Athens, Athens, Greece \\ Email: aprob@teiath.gr
}

Received 23 January 2013; revised 15 February 2013; accepted 14 March 2013

Copyright (C) 2013 Anthony E. Prombonas et al. This is an open access article distributed under the Creative Commons Attribution License, which permits unrestricted use, distribution, and reproduction in any medium, provided the original work is properly cited.

\begin{abstract}
The aim of this research is to clarify whether the midline axis of the complete upper denture (CUD) is the axis of its torsion during loading, apart from the known bending deflection. Furthermore the present study is intended to estimate the influence of the anterior notches on the torsional deformation of the CUD. Using commercial edentulous molds and standardized procedures, six identical CUDs were fabricated with an initial fraenal notch of $5 \mathrm{~mm}$. Two additional notch conditions were produced by deepening the notch to a total depth of $9 \mathrm{~mm}$ and by creating an incisal diastema of $7 \mathrm{~mm}$. Five biaxial (also known as "fish bone") strain gauges were cemented onto the palatal section of the dentures so that their middle axis coincided with the midline axis of the outer surface of the denture. For the denture specimen used in this study, the specific point of torsion was detected 2 cm from the point of contact of the two artificial central incisors. The presence of a deep fraenal notch and the combination of the deep fraenal notch with the presence of an incisal diastema increased the torsion of the CUD to a significant level $(P=0.006$ and $P$ $=0.05$, respectively). It was shown that the midline can be the CUD's axis of torsion. Biaxial strain gauges could be a reliable method of measuring the torsional deformation of the CUD.
\end{abstract}

Keywords: Complete Upper Denture; Torsional Deformation; Biaxial Strain Gauge

\section{INTRODUCTION}

The fracture of the acrylic resin dentures is an unresolved problem in removable prosthodontics despite numerous attempts to determine its cause. Despite the high frequency of complete upper denture (CUD) fracture, there is surprising little discussion of the subject in the literature. Nevertheless it has been established, that the CUDs are subjected to bending deformation [1].

The above consideration leads to the reasonable assumption that there may be a deficit in the study of the mechanical behavior of the CUD in functional loading conditions. The above assumption in combination with the fact that the corresponding research has focused on the study of pure bending of the CUD, may be the reason why mechanical behavior of the CUD still remains a complicated problem.

Recent studies have shown that the complexity of the loads applied during the normal function of the CUD, in both magnitude and direction, and the alterations in the directions and magnitudes of principal stresses induce a complex multiaxial stress state in the palatal stress field and in the field of the CUD's labial flange [2-4].

Engineered mechanical structures, such as dentures, can be subjected to much more complex systems of loading [5,6]. Multiaxial fatigue damage occurs when the principal stress directions vary. Moreover real loads can induce bending combined with torsional, axial and shear stresses, generating bi- or tri-axial variable stress/strain histories at the critical point and leading to multiaxial fatigue problems [7-10].

Bearing in mind all the previous views, it is reasonable to question whether the loaded CUD only bends on the midline of the palate or whether a combination of bending and torsion of the denture may occur.

There is currently no published research related to the issue of torsion in the CUD. For this reason, relevant articles in the engineering literature have been examined. For the simplification of the problem, the assumption could be made that the torsional deformation of the CUD can be studied through the torsional deformation of a very thin and wide beam (actually a plate) with free ends. Torsional stresses are introduced when the applied load tends to twist the beam. This will occur when a beam supports 
a load that is applied eccentric to the principal crosssectional axis $[11,12]$.

The present study is intended to gain additional information over the torsional deformation of the CUD, aiming to supplement the ascertained deficit in the mechanical behavior of the CUD. Since the bending deformation of the CUD has been studied extensively in the past, this pilot investigation was initiated to examine only the mean shear stresses recorded on the midline axis of the CUD and clarify if the midline axis of the denture is the axis of its torsion during loading. Other aims of this work include determining where the torsion point is located on the axis of the CUD and estimating the influence of notches on the anterior segment of the denture on its torsional behavior.

\section{MATERIALS AND METHODS}

\subsection{Construction of the Identical Dentures-Specimens}

Six identical CUDs were constructed according to a previous methodology, which is briefly described below [24].

Two commercial molds of edentulous jaws were used: one of the upper jaw and one of the lower jaw (edentulous molds, size 55; Columbia Dentoform, Long Island, New York, USA) $[2,3,13]$.

These molds were used to fabricate two prototype casts from type III dental stone (Hydrock, KerrLab, Orange, California, USA). Using a standardized methodology, two wax denture bases of uniform thickness (2.5 $\mathrm{mm}$ ) were constructed using wax denture sheets (Tenatex, Associated Dental Products, Wiltshire, UK). Waxed rims were placed on the denture bases so that their long axes coincided with the crests of the residual ridges. Guidelines were drawn on the waxed rims to indicate the position of the ridge crest. The anterior teeth (Upper Anterior, Uhler Dental Supply, Chicago, Illinois, USA) were arranged according to standard procedures $[2,3,13,14]$.

The posterior teeth (Upper Posterior, $33^{\circ}$; Uhler Dental Supply) were placed with the central grooves of their occlusal surfaces coinciding with the guidelines, and the occlusal surfaces were arranged according to standard procedures to reproduce the anteroposterior and lateral compensating curves $[13,14]$.

The corresponding wax complete lower denture (CLD) was then constructed with standardized vertical dimensions of occlusion using the corresponding lower teeth (Lower Posterior, $33^{\circ}$; Uhler Dental Supply).

The waxed CUD was removed from the upper cast of the articulator and used to construct a two-piece mold to reproduce six identical standardized CUDs. A new cast was placed in its respective position in the lower half of the mold (made from Plexiglas), while the upper half of the mold (made with silicone) was fixed in position on the lower half (made from Plexiglas). Then, molten baseplate wax (Tenatex, Associated Dental Products) was poured into the intervening space. This procedure yielded six fully waxed CUDs and a waxed CLD, which were then flasked using an acrylic-resin material in accordance with the manufacturer's instructions (Paladon 65, Heraeus Kulzer, Hanau, Germany). Thus, six identical CUDs were obtained with teeth made from the same material as was used to construct the denture bases. Artificial teeth were not used for the construction of the identical dentures specimens because if this was the case, the artificial teeth could be placed at a slightly different location, resulting in different loading condition of the acrylic dentures bases. The acrylic dentures were finished according to standard finishing procedures for acrylic-resin denture bases [14].

During the grinding and polishing of the definitive acrylic-resin dentures, the thickness was measured at six points on each of the labial and buccal flanges using an analog thickness gauge with $0.1 \mathrm{~mm}$ precision (K series, Schmidt Control Instruments, Waldkraiburg, Germany) to ensure that the bases of all of the denture specimens had the same thickness $(3.0 \pm 0.1 \mathrm{~mm})[2-4]$.

\subsection{Strain Gauges}

It is a common view of many researchers that strain gauges are a reliable method for measuring torque $[15$, 16].

According to the simple theory of torsion on isotropic rectangular cross-section beams, the principal direct strains $\left(\varepsilon_{1}, \varepsilon_{2}\right)$ are inclined at $\pm 45^{\circ}$ to the torsion axis (the longitudinal center line) and are equal in magnitude but opposite in sign $[10,12]$. In this case the following equations are valid:

$$
\begin{gathered}
\varepsilon_{1,2}=\frac{t}{2 L} \theta \\
T=G \beta w t^{2} 2 \varepsilon_{1,2}
\end{gathered}
$$

where $T$ is the torque, $t$ is the specimen thickness, $L$ is the length of the specimen, $\theta$ is the angle of twist, $G$ is the shear modulus, $w$ is the width of the specimen, and $\beta$ is a known coefficient dependent on the thickness and width of the specimen $[10,12]$.

If a torsion axis exists on the structure, for example the CUD, then by affixing biaxial strain gauges with their sensitive axes oriented at $\pm 45^{\circ}$ to the longitudinal axis of the structure, it is possible to record the shear strains due to the torsion $[11,16]$.

When a biaxial two elements (also known as "torsion" or "fish bone") strain gauge is cemented on a specimen which is under torsion, such that its central axis coincided to the axis of torsion and the gridlines of its one 
element are at $45^{\circ}$ to this axis, the bridge output is sensitive to the torque but insensitive to any bending or axial loads (Figure 1) [17].

By connecting the two elements of the biaxial strain gauge to a half-bridge circuit it can be shown through mathematical equations that the shearing strain $\gamma_{\mathrm{xy}}$ can be measured. The function $\varepsilon_{1}-\varepsilon_{2}$ will be performed automatically in the bridge, and the output will yield $2 \gamma_{\mathrm{xy}}$ directly [17].

Five biaxial strain gauges for torsion measurement (type KFG-2-120-D31, Kyowa Electronic Instruments, Tokyo, Japan) were cemented onto each identical upper denture with cyanoacrylate adhesive (type CC-15A, Kyowa Electronic Instruments). The strain gauges were cemented onto the palatal section of the dentures so that their middle axis coincided with the midline axis of the outer surface of the denture. Their centers were positioned at points that were $1,2,3,4$, and $5 \mathrm{~cm}$ from the contact point of the central incisors (Figure 2). All of the strains were measured using an electronic six-channel data acquisition system (Wheatstone bridge) consisting of a personal computer, a 12 bit PCI A/D card, a terminal board and specific acquisition and control software (Advanced GeniDAQ; American Advantech Corporation, Sunnyvale, California, USA). The three wires of each individual torque gauge were connected to the electronic Wheatstone bridge to produce a two active half-bridge connection for each gauge, which resulted in five halfbridge circuits.

Each upper denture specimen and the selected lower denture were mounted onto die stone casts, made by pouring high-strength type IV dental stone (Vel-Mix, KerrLab, Orange, California, USA) into the two commercial jaw molds, using the methodology of previous studies [2-4, 13].

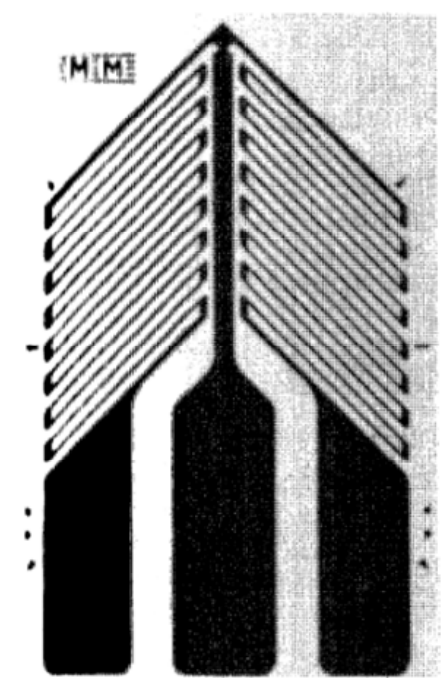

Figure 1. The biaxial (also known as fish bone) strain gauge.

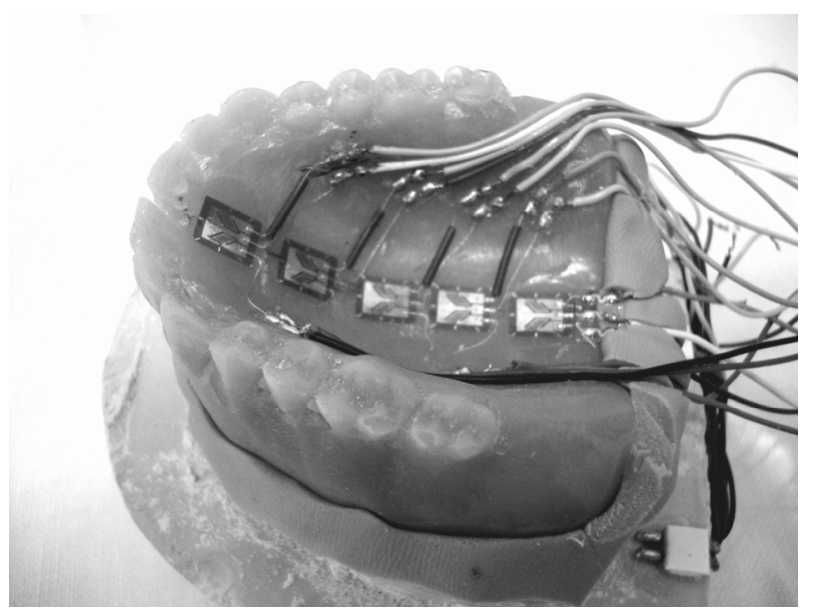

Figure 2. The arrangement of the five biaxial strain gauges on the midline of the palate of the CUD.

Before the denture specimens were placed on the load casts, the intaglio surfaces were painted with mediumviscosity silicone (Coltoflax, Coltene/Whaledent, NJ, USA) to simulate the oral mucosa. To ensure that the thickness of the silicone was similar to the average thickness of the masticatory mucosa in vivo, the thicknesses of the die stone were reduced by $2 \mathrm{~mm}$ and $1.5 \mathrm{~mm}$ in the residual ridges of the maxillary cast and the mandibular cast, respectively, before the placement of the painted specimens $[2-4,13]$.

The upper and lower dentures were positioned in centric occlusion, and their mounted casts were placed between the plates of a hydraulic press (Bego Hydraulic, BEGO, Bremen, Germany) (Figure 3).

Each denture specimen was loaded three times under three notch conditions: condition 1 was loaded with the initial fraenal notch (5 $\mathrm{mm}$ in depth), condition 2 was loaded after deepening the notch by $4 \mathrm{~mm}$ (to a total notch length of $9 \mathrm{~mm}$ ) using a cutting wheel (Ceraflex high-cutting-efficiency wheel, Bredent, Germany), and condition 3 was loaded after creating an incisal (midline) diastema by making a $7 \mathrm{~mm}$ slot between the two central incisors. This latter condition was considered as representative of the creation of a diastema between the teeth at request of the patient or of the presence of a crack due to insufficient penetration of acrylic resin between the teeth. The loads were applied at time intervals of 2 hours in duration to allow the acrylic base to recover from the previous load $[4,18]$.

The denture specimens were loaded at $100 \mathrm{~N}$. The maximum load of $100 \mathrm{~N}$ is consistent with a previous study that showed that the force exerted by edentulous patients at the vertical dimension of occlusion ranges from 30 to $110 \mathrm{~N}$ [19].

The shear strain was determined for every denture specimen and for each of the three notch conditions. The final mean value of the stress was determined from the 


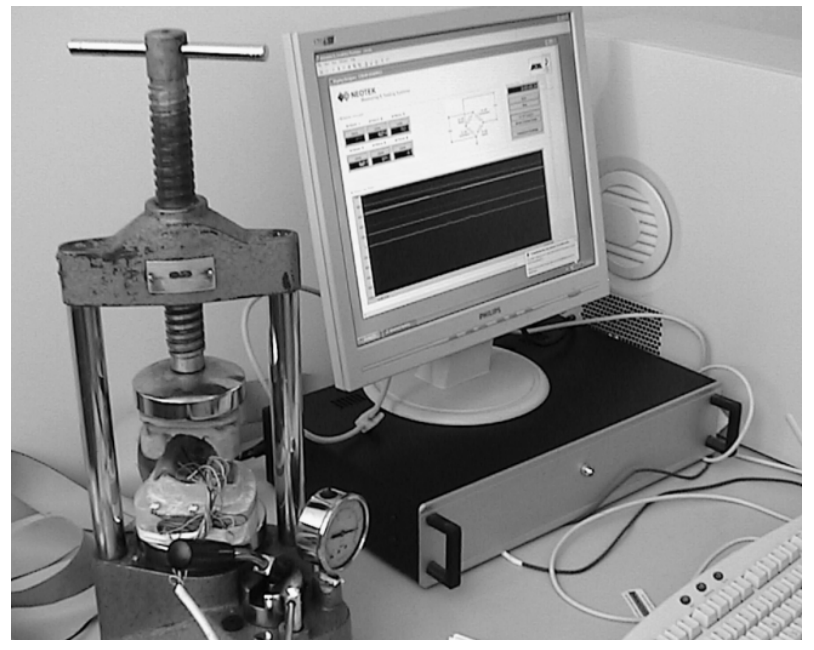

Figure 3. The CUD mounted on the cast in centric occlusion with the antagonistic lower denture, between the plates of the press.

six identical complete-denture specimens and was considered to be the representative value of the strain for every strain gauge in the corresponding notch condition.

The sensitivity of the used biaxial strain gauge in torsion, was measured by cementing a gauge onto an acrylic plate with dimensions of $6 \mathrm{~cm} \times 3 \mathrm{~cm} \times 3 \mathrm{~mm}$ so that its long axis coincided with the middle axis of the long dimension of the plate.

One end of the plate, which could be considered as a wide rectangular beam, was clamped to a bench. By applying a known torque of $1.2 \mathrm{Nm}$ on the other end of the plate (free end) deflections in three orientations were induced, allowing three strains to be measured on the plate.

The orientations producing bending along the long axis of the plate and along the short axis of the plate (with no torsional deflection) induced recorded strains of 215 and $288 \mu$ Strains, respectively. The strain recorded for the third orientation (rotation around the long axis of the plate causing pure torsional deflection) was $3830 \mu$ Strains. From these results it can be concluded that strain values of up to $300 \mu$ Strains, during the loading of the denture specimens, indicate deformations resulting from bending without torsional deformation, while values exceeding $300 \mu$ Strains indicate small to large torsional deformations.

\subsection{Statistical Analysis}

One-way ANOVA was applied using standard statistical software (SPSS version 15.0 for Windows, SPSS, Chicago, Illinois, USA) to estimate the statistical significance of the differences between the mean values of shear strains in the three notch conditions. In addition, a Tukey HSD test was used for multiple comparisons among all possible pairs of the three notch conditions. The significance level was set as $P=0.05$.

\section{RESULTS}

Figure 4 is a graphical representation of the mean shear strains recorded by each of the strain gauges in the three notch conditions. In this figure, position 1 refers to the anterior palatal strain gauge, position 5 refers to the posterior palatal strain gauge, and positions 2, 3 and 4 refer to the intervening strain gauges (Figure 4). As can be seen in this figure, the mean values of the shear strains for gauges 1, 3, 4 and 5 are low enough, lower than the threshold value of $300 \mu$ Strains to be safely considered as deformations caused by bending. For this reason, the deformations (mean values) recorded by strain gauges 1 , 3,4 and 5 are excluded from further evaluation because these deformations may not be due to torsion. Consequently, the statistical analysis mentioned above was performed only for the strains measured on strain gauge 2 .

Figure 5 is a representation of the mean shear strains measured by the strain gauge of position 2 . The results for this strain gauge indicate that its deformations are all greater than $1000 \mu$ Strains (Figure 5). More precisely, for the initial fraenum (condition 1), the shear strain was $1025.3 \mu$ Strains; for the deep fraenal notch (condition 2), the strain was $1934.6 \mu$ Strains; and for the combination of the deep fraenal notch and the incisal diastema (condition 3), the strain was $1545.1 \mu$ Strains. According to measurements made on the sensitivity of the biaxial strain gauge, all the above deformations could safely be considered as deformations caused by torsion.

The mean values of the strains for the three notch conditions varied in a statistically significant manner $(P=$ 0.006). The pair wise comparison of the three notch conditions shows that condition 1 (initial fraenum) was statistically significantly different from conditions 2 and 3 (the deep fraenal notch and a combination of the deep fraenal notch with an incisal diastema, respectively) $(P=$

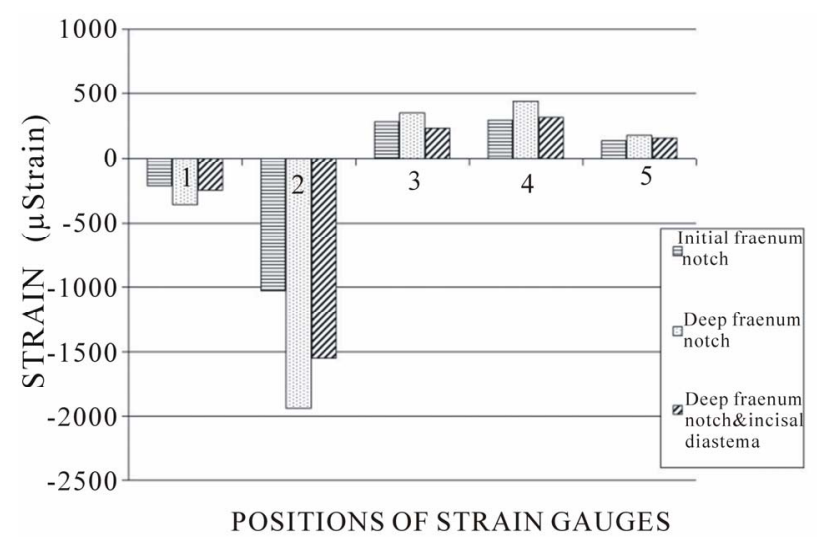

Figure 4. The recorded strains, on the five biaxial strain gauges for the three notch conditions. 


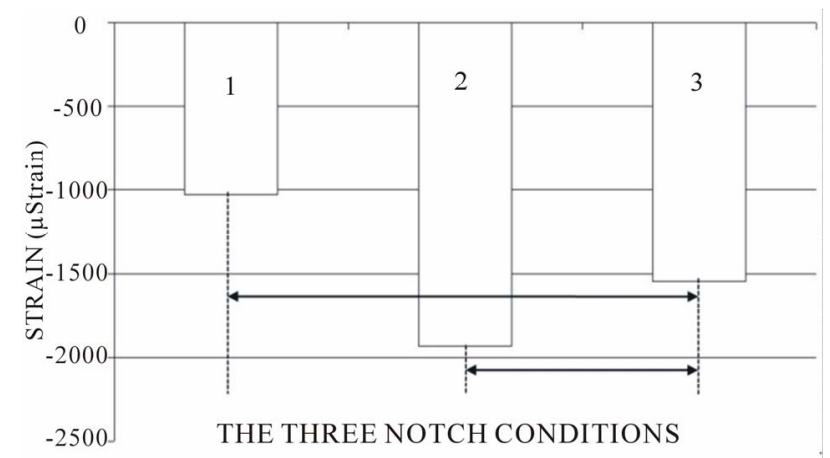

Figure 5. The recorded strains on the strain gauge of position 2, in the three notch conditions. The horizontal lines connect the notch conditions with mean strains that are different in a statistically significant level.

0.006 and $P=0.05$, respectively), while the difference between conditions 2 and 3 was not statistically significant $(P=0.52)$.

\section{DISCUSSION}

A range of studies have shown that during the loading of dentures, a multiaxial state of stress develops on the palatal region [2-4,14]. It has also been suggested, from studies on mechanical constructions, that torsion could coexist with bending during a multiaxial stress situation [7-10].

Thus, it can be assumed that the midline of the palate may be the torsion axis of the CUD during loading.

In the present investigation, using biaxial strain gauges cemented on the midline of the palate it was shown that the initial assumption was correct; the point of torsion is located $2 \mathrm{~cm}$ from the point of contact of the two artificial central incisors (the strain gauge of position 2) (Figure 6).

It was also shown that the presence of notches on the anterior portion of the CUD (the fraenal notch and an incisal diastema) significantly increased the torsion (oneway ANOVA, $P=0.006$ ). Among the three notch conditions tested, only two of them, the presence of a deep fraenal (labial) notch and the deep fraenal notch in combination with an incisal diastema, increased the torsion of the CUD to a significant level $(P=0.006$ and $P=0.05$, respectively).

Further research is needed to investigate several issues, such as the factors affecting the location of the point of torsion on the midline of the CUD and the influence of the shape and size of the palatal vault of the CUD on its torsional deformation.

It is commonly acknowledged that the problem of the flexural deformation of the CUD is complex and intractable $[1-4,14,15]$. A possible explanation for this may be that a multiaxial state of stress develops during the loading of the CUD and it may be subjected to torsion around

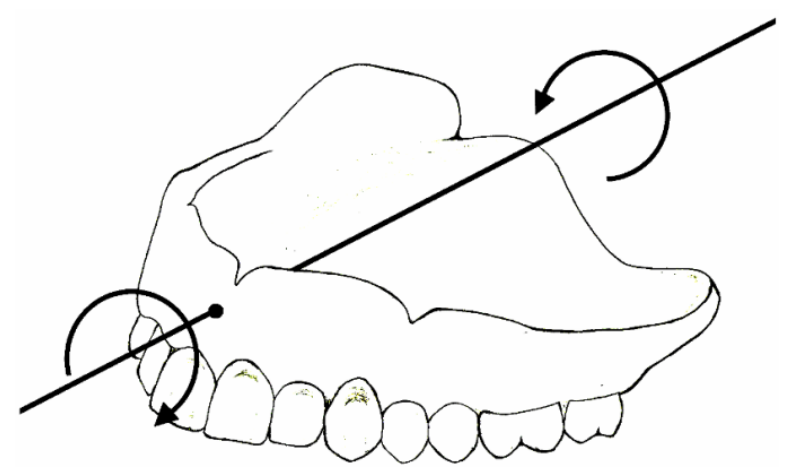

Figure 6. A graphical representation of the torsional deformation of the CUD.

its palate midline axis, apart from the known bending deflection, as shown by the present research.

\section{CONCLUSIONS}

Based on the results of present study and within the limitations resulting from the applied methodology, the following conclusions can be formulated.

The midline of the palate of the CUD could be the torsion axis of the denture during loading.

For the denture specimens used in this study, the point of torsion was detected $2 \mathrm{~cm}$ from the point of contact of the two central artificial incisors on the midline axis of the palatal region of the CUD.

The presence of notches on the anterior region of the denture (a fraenal notch and an incisal diastema) significantly increased the torsion of the CUD.

The assumption of the torsional deformation of the CUD during function in coexistence with the bending deflection, may lead to the understanding of the integrated mechanical behavior of the CUD, aimed at more reliable functional dentures.

\section{REFERENCES}

[1] Beyli, M.S. and Fraunhofer, J.A. (1981) An analysis of causes of fracture of acrylic resin dentures. Journal of Prosthetic Dentistry, 46, 238-241. doi:10.1016/0022-3913(81)90206-7

[2] Prombonas, A. and Vlissidis, D. (2002) Effects of the position of artificial teeth and load levels on the stress in the complete maxillary denture. Journal of Prosthetic Dentistry, 8, 415-422. doi:10.1067/mpr.2002.128174

[3] Prombonas, A. and Vlissidis, D. (2009) Analysis of stresses in complete upper dentures with flat teeth at differing inclinations. Medical Engineering and Physics, 31, 314319. doi:10.1016/j.medengphy.2008.06.008

[4] Prombonas, A., Vlissidis, D., Paralika, M.A. and Poulis, N.A. (2012) The stress state of the fraenal notch region in the complete upper dentures. Medical Engineering and Physics, 34, 1477-1482. 
doi:10.1016/j.medengphy.2012.02.009

[5] Taylor, D. (2007) The theory of critical distances. A new perspective in fracture mechanics. Elsevier, London.

[6] Smyd, E.S. (1961) The role of torque, torsion and bending in prosthetic failures. Journal of Prosthetic Dentistry, 11, 95-111. doi:10.1016/0022-3913(61)90116-0

[7] Liu, Y. and Mahadevan, S. (2007) A unified multiaxial fatigue damage model for isotropic and anisotropic materials. International Journal of Fatigue, 29, 347-359. doi:10.1016/j.ijfatigue.2006.03.011

[8] Wang, Y.Y. and Yao, W.X. (2006) A multiaxial fatigue criterion for various metallic materials under proportional and nonproportional loading. International Journal of $\mathrm{Fa}$ tigue, 28, 401-408. doi:10.1016/j.ijfatigue.2005.07.007

[9] Ahmadi, A. and Zenner, H. (2006) Lifetime simulation under multiaxial random loading with regard to the microcrack growth. International Journal of Fatigue, 28, 954-962. doi:10.1016/j.ijfatigue.2005.09.015

[10] Meggiolaro, M.A., Castro, J.T.P. and Miranda, A.C.O. (2009) Evaluation of multiaxial stress-strain models and fatigue life prediction methods under proportional loading. Proceedings of the Second International Symposium on Solid Mechanics, Rio De Janeiro, 28-30 April 2009, 365-384.

[11] Timoshenko, S.P. and Goodier, J.N. (1981) Theory of elasticity. McGraw-Hill, New York.

[12] Cordey, J. (2000) Introduction: Basic concepts and defi- nitions in mechanics. Injury, 31, 1-13. doi:10.1016/S0020-1383(00)80039-X

[13] Ravi, N., Krishna, D.P., Manoj, S. and Chethan, H. (2010) A functional stress analysis in the maxillary complete denture influenced by the position of artificial teeth and load levels: An in-vitro study. Journal of Indian Prosthodontic Society, 10, 219-225. doi:10.1007/s13191-011-0046-0

[14] Zarb, G.A., Bolender, C.L. and Carlsson, G.E. (1997) Bouchers's prosthetic treatment for edentulous patients. Mosby, St. Louis.

[15] Muftah, M.H. and Haris, S.M. (2011) A strain gauge based system for measuring dynamic loading on a rotating shaft. International Journal of Mechanics, 5, 19-26.

[16] Turvey, G.J. (1998) Torsion tests on pultruded GRP sheet. Composites Science and Technology, 58, 1343-1351. doi:10.1016/S0266-3538(98)00003-7

[17] Dally, J. and Riley, W. (1991) Experimental stress analysis. College House Enterprises LLC, Knoxville.

[18] Sorensen, R.E. and Ryge, G. (1962) Flow and recovery of denture plastics. Journal of Prosthetic Dentistry, 12, 10791088. doi:10.1016/0022-3913(62)90163-4

[19] Prombonas, A., Vlissidis, D. and Molyvdas, P. (1994) The effect of altering the vertical dimension of occlusion on biting force. Journal of Prosthetic Dentistry, 71, 139143. doi:10.1016/0022-3913(94)90021-3 